Bio - grafia. Escritos sobre la Biología y su Enseñanza. ISSN 2027

Edición Extraordinaria. p.p. 513 - 520

Memorias del VIII Encuentro Nacional de Experiencias en Enseñanza de la Biología y la Educación Ambiental. III Congreso Nacional de Investigación en Enseñanza de la Biología.

\title{
HISTORIA Y FILOSOFÍA DE LA CIENCIA Y SUS CONTRIBUCIONES PARA LA EDUCACIÓN CIENTÍFICA Y MATEMÁTICA: UN ESTUDIO EN PERIODICOS BRASILEROS DEL ÁREA DE ENSEÑANZA
}

\author{
HISTORY AND PHILOSOPHY OF SCIENCE AND THEIR CONTRIBUTIONS TO \\ SCIENTIFIC AND MATHEMATICAL EDUCATION: A STUDY OF BRAZILIAN \\ JOURNALS IN THE FIELD OF EDUCATION
}

\author{
Simone Luccas ${ }^{1}$ \\ Rudolph dos Santos Gomes Pereira ${ }^{2}$ \\ Lucken Bueno Lucas ${ }^{3}$ \\ Rodrigo de Souza Poletto 4 \\ Annecy Tojeiro Giordani ${ }^{5}$
}

Resumen: La aproximación entre las áreas de Historia, Filosofía, Matemática y las Ciencias Naturales ha sido bien aceptada no solamente en el ambiente de las investigaciones científicas sino también en el campo educativo, como una alternativa didáctica para la enseñanza del conocimiento científico y matemático. Con todo, se hace necesario comprender como esta aproximación se llevó a cabo. Así, el objetivo de este estudio fue analizar de qué modo las áreas específicas de Biología, Ciencias, Física, Química y Matemática han lidiado con esta aproximación. Por tanto, se emprendió una investigación en tres periódicos brasileros, clasificados en el sistema webqualis como A1 (uno). Los datos recolectados fueron analizados a la luz del Análisis de Contenido, según Bardin. A Partir del análisis fue posible inferir que la cantidad que investigaciones publicadas, relativas a la inserción de los estudios de Historia y Filosofía de la Ciencia en la enseñanza de las

1 Profesora del Departamento de Matemáticas - Universidad Estadual do Norte do Paraná (UENP), Brasil. Correo electrónico: simoneluccas@uenp.edu.br;

${ }^{2}$ Profesor del Departamento de Matemáticas - Universidad Estadual do Norte do Paraná (UENP), Brasil. Correo electrónico: rudolphsantos@uenp.edu.br

${ }_{3}^{3}$ Profesor del Departamento de Biología - Universidad Estadual do Norte do Paraná (UENP), Brasil. Correo electrónico: luckenlucas@uenp.edu.br;

${ }^{4}$ Profesor del Departamento de Biología - Universidad Estadual do Norte do Paraná (UENP), Brasil. Correo electrónico: rodrigopoletto@uenp.edu.br;

${ }^{5}$ Profesora del Departamento de Enfermería - Universidad Estadual do Norte do Paraná (UENP), Brasil. Correo electrónico: annecy@uenp.edu.br. 
Bio - grafia. Escritos sobre la Biología y su Enseñanza. ISSN 2027

Edición Extraordinaria. p.p. 513 - 520

Memorias del VIII Encuentro Nacional de Experiencias en Enseñanza de la Biología y la Educación Ambiental. III Congreso Nacional de Investigación en Enseñanza de la Biología.

Ciencias y Matemáticas es pequeña en comparación a la cantidad total de investigaciones realizadas.

Palabras Clave: Historia y Filosofía de la Ciencia, Enseñanza de las Ciencias Naturales, Enseñanza de la Matemática.

Abstract: The closeness between the fields of History, Philosophy and the Natural Sciences, including Mathematics, has been widely accepted not only in the context of scientific research, but in the educational field, as a didactic alternative to the teaching of scientific and mathematical knowledge. However, it is necessary to understand how this convergence occurred. Thus, the objective of this study was to analyze the manner in which specific areas of Biology, Science, Physics, Chemistry and Mathematics have been dealing with this approach. To this effect, an investigation was undertaken in three Brazilian journals, classified as $A 1$ in the webqualis system. The data collected were analyzed based on Content Analysis, according to Bardin. From the analysis, it was possible to infer that the quantity of published research, regarding the insertion of History and Philosophy of Science studies into Science and Mathematics Teaching was scarce when compared to the total quantity of research conducted.

Key-words: History and Philosophy of Sciences; Teaching Natural Sciences; Teaching Mathematics.

\section{Introducción}

Diversas investigaciones han evidenciado una aproximación tanto de la Historia como de la Filosofía de la Ciencia con la Educación Científica, a partir de las áreas de la Biología, Ciencias, Física, Química y Matemática (Matthews (1995), Wortmann (1996), Maienschein (2000), El-Hani, Tavares \& Da Rocha (2004), Luccas (2004) e Delizoicov, Angotti \& Pernambuco (2007), Lucas (2010), entre otros). Con todo, existe un aspecto que debe analizarse y toca el modo como esa aproximación ha sucedido en el ámbito educacional, teniendo en vista que hay reflexiones que son necesarias cuando se utilizan fundamentos filosóficos en la enseñanza del conocimiento científico.

Los aportes de la Historia y de la Filosofía de la Ciencia (HFC), según Matthews (1995), son reveladores para los procesos de enseñanza y aprendizaje de una manera general, teniendo 
Bio - grafia. Escritos sobre la Biología y su Enseñanza. ISSN 2027

Edición Extraordinaria. p.p. 513 - 520

Memorias del VIII Encuentro Nacional de Experiencias en Enseñanza de la Biología y la Educación Ambiental. III Congreso Nacional de Investigación en Enseñanza de la Biología.

en cuenta que actualmente existe una crisis en la enseñanza. Aunque el investigador, unida a esta crisis ha ocurrido una evasión en el ámbito educacional, no solamente por parte de los alumnos, sino también por parte de los profesores.

Uno de los responsables por este contexto socio-científico, segundo Matthews (1995), puede ser el hecho de que la enseñanza de las ciencias se ha desarrollado totalmente separada de la Historia y de la Filosofía. Con todo esto, autor comenta que a partir de la década de los ochenta ha sucedido una reaproximación entre tales áreas.

Factores positivos, ocasionados por esta aproximación (HFC), han sido dirigidos en diversas investigaciones, pues ella hace con que las clases se tornen más reflexivas y estimulantes, además de ayudar a desarrollar el pensamiento crítico; contribuye para la humanización de las ciencias; posibilita una compresión más integral del conocimiento científico (por parte de los maestros) disminuyendo la falta de significado y la compresión epistemológica de la Ciencia, llevándolos consecuentemente, a mejorar su práctica docente (Matthews, 1995). Con relación a este último aspecto, Wortmann (1996) destaca las investigaciones más fructuosas parecen ser aquellas que se disponen a promover el análisis del contenido conceptual sobre el punto de vista histórico-epistemológico, sin dejar de considerar el papel del estudiante en la construcción de su propio saber. El análisis del contenido de los conceptos propios de una área específica del saber, tienen un importante papel en el alcance de una comprensión más profundada acerca del conocimiento propio de la misma área. Además de eso, este procedimiento envuelve, necesariamente una interrelación interdisciplinar capaz de fortalecer el propio dominio disciplinar, en la medida en que se favorece el esclarecimiento de los aspectos referentes a la organización del saber, para explicar las relaciones entre los diversos "elementos" de ese saber. (p. 70)

Maienschein (2000) observa los aspectos positivos de la aproximación entre la Ciencias, la Historia y la Filosofía, pues en su análisis ellas ayudan a comprender y hacer Ciencia de un modo mejor, librando profesores e alumnos de las perspectivas limitadas.

Entre tanto, teniendo en consideración los diversos aspectos presentados por los investigadores nombrados anteriormente, se suscita una interrogación: ¿De qué manera ha ocurrido la aproximación entre estas áreas? 
Bio - grafia. Escritos sobre la Biología y su Enseñanza. ISSN 2027

Edición Extraordinaria. p.p. 513 - 520

Memorias del VIII Encuentro Nacional de Experiencias en Enseñanza de la Biología y la Educación Ambiental. III Congreso Nacional de Investigación en Enseñanza de la Biología.

Así, el objetivo de esta investigación es comprender la manera como las áreas específicas de la Biología, la Ciencia, la Física, la Química y la Matemática han lidiado con esta aproximación.

\section{Metodología}

Los datos arrojados por esta investigación fueron analizados a la luz del Análisis de Contenido, que según Bardin (2011) comprende un conjunto de técnicas de investigación realizado en las comunicaciones con el objetivo de analizar de forma objetiva y sistemática sus mensajes, por medio de indicadores cuantitativos o cualitativos que posibiliten interpretar e inferir conocimientos relativos a esas comunicaciones.

Para conseguir el objetivo de este estudio, fue realizada una investigación en periódicos nacionales de Brasil, clasificados como $A 1$ en el sistema webqualis, según a CAPES Comissão de Aperfeiçoamento de Pessoal do Nível Superior (Comisión de Perfeccionamiento de Personal a Nivel Superior). En este sentido fueron encontrados tres periódicos nacionales a saber: Ciência \& Educação (Ciencia y Educación), Bolema e Revista Brasileira de Ensino de Física (Bolema y Revista Brasilera de Educación Física). La investigación fue realizada en los volúmenes publicados entre los años 2005 y 2015. En cuanto a los criterios adoptados para la selección de los artículos, primeramente fueron analizados los títulos de los mismos que presentaran relación con las áreas de Historia o Filosofía de las Ciencias Naturales - incluyendo la Matemática. En un segundo momento, fueron analizadas las palabras clave y, en caso de dudas, los resúmenes fueron leídos.

En el periodo de investigación fueron analizados 1.623 artículos, de los cuales solamente 134 desarrollaban la temática exigida por el estudio.

\section{Resultados y discusiones}

A partir de este análisis fueron configuradas diez categorías, en las cuales los artículos fueron clasificados. La cantidad y el porcentaje de frecuencia en relación al total de los artículos seleccionados se encuentran en el cuadro 1.

Cuadro 1: categorías establecidas 
Bio - grafia. Escritos sobre la Biología y su Enseñanza. ISSN 2027

Edición Extraordinaria. p.p. 513 - 520

Memorias del VIII Encuentro Nacional de Experiencias en Enseñanza de la Biología y la Educación Ambiental. III Congreso Nacional de Investigación en Enseñanza de la Biología.

\section{CATEGORÍAS}

Uso de la Historia enseñanza de las Ciencias

Uso de la Filosofía o Epistemología en la enseñanza de las

Ciencias.

Estudio de la Historia de la Filosofía en la enseñanza de las

Ciencias

Estudio histórico-conceptual en la enseñanza de las Ciencias

Estudio de las relaciones entre la Historia, la Filosofía y las

Ciencias

Historia de las Ciencias

Estudio de la Historia de conocimientos de Ciencia

Estudio de la Filosofía/Epistemología de conocimientos da

Ciencia

Estudio de las relaciones entre Historia, Filosofía y Didáctica

Estudios histórico/sociológicos de las Ciencias

Fuente: De los autores

$\begin{array}{cc}\text { CANTIDAD } & \text { PORCENTA } \\ \text { DE } & \text { JE (\%) } \\ \text { ARTICULO } & \\ \text { S } & \\ 13 & 9,70 \\ 6 & 4,48 \\ & \\ 6 & 4,48 \\ & \\ 2 & 1,49 \\ 3 & 2,24 \\ & \\ 21 & 15,67 \\ 60 & 44,78 \\ 12 & 8,96 \\ & \\ 9 & 6,72 \\ 2 & 1,49 \\ 134 & 100\end{array}$

TOTAL 134
100

Como es posible notar en estos datos, aún es poca la cantidad de investigaciones realizadas, en el ámbito brasilero, relativas a la inserción de los estudios de Historia y Filosofía de las Ciencias Naturales y Matemática en el enseñanza de estas áreas de conocimiento, teniendo en cuenta que del total de 1.623 artículos existentes en estas tres revistas, en los últimos diez años solamente 134 o sea, el $8,26 \%$ de las investigaciones existentes, envuelven la relación entre estas áreas del conocimiento científico.

Cabe resaltar también que hay una cantidad mayor de investigaciones relacionadas a la Historia y a las Ciencias, en detrimento a las relativas a la Filosofía y las Ciencias. De las 134 investigaciones encontradas, 9,7\% aborda investigaciones que discuten el uso de la Historia en la enseñanza de las Ciencias, el 15,67\% trata de las Historia de las Ciencias y un $44,78 \%$ aborda estudios históricos que tratan del desarrollo de conocimientos específicos de las Ciencias y, totalizando, el 70,15\% de los artículos encontrados trata exclusivamente de la Historia. 
Bio - grafia. Escritos sobre la Biología y su Enseñanza. ISSN 2027

Edición Extraordinaria. p.p. 513 - 520

Memorias del VIII Encuentro Nacional de Experiencias en Enseñanza de la Biología y la Educación Ambiental. III Congreso Nacional de Investigación en Enseñanza de la Biología.

Pocos estudios discuten aspectos filosóficos o aún más epistemológicos de las Ciencias, menos incluso se discute la inserción de las investigaciones científicas en el ámbito social. No se puede dejar de lado que el $29,85 \%$ de los artículos encontrados está relacionado con esa temática.

Es posible inferir que la perspectiva de muchas investigaciones científicas brasileras presentes en las revistas analizadas se restringe a un contexto bien especifico y en este sentido, hay una compresión por parte de la comunidad científica de que si tales investigaciones fueran desarrolladas y analizadas en un contexto más amplio, según perspectivas histórico filosóficas, podría conducir a los investigadores a una ampliación de la visión de sus estudios mediante el contexto socio histórico-cultural contemporáneo (Matthews, 1995) y también contribuir para una formación académica más efectiva (Maienschein, 2000), una vez que posibilitara a los iniciantes a aprehender una visión más dinámica y amplia de los conocimientos específicos estudiados en las Ciencias (Wortmann, 1996).

\section{Consideraciones finales}

La aproximación realizada entre la Historia, la Filosofía, las Ciencias Naturales y la Matemática se ha presentado con éxito en el ambiente de las investigaciones científicas como una alternativa pedagógica que contribuye al proceso de construcción del conocimiento.

Una de las principales contribuciones surge en la cuestión de que esta aproximación posibilita una compresión de las Ciencias no como listas y culminadas, sino como desarrollos humanos realizados a lo largo del tiempo, con estudiosos que anduvieron por caminos algunas veces correctos otras veces no tanto, pero siempre buscando entender mejor el mundo.

Además de esa compresión, el hecho de trabajar en esa aproximación entre la Historia y la Filosofía en la enseñanza de las Ciencias permite tener a los alumnos una visión diferenciada de estas áreas del conocimiento, una vez que estas les ofrecen una perspectiva humana de la sistematización de las Ciencias, pues previendo subsidios para que los alumnos puedan verlas, no como áreas estáticas que cargan informaciones sobre el 
Bio - grafia. Escritos sobre la Biología y su Enseñanza. ISSN 2027

Edición Extraordinaria. p.p. 513 - 520

Memorias del VIII Encuentro Nacional de Experiencias en Enseñanza de la Biología y la Educación Ambiental. III Congreso Nacional de Investigación en Enseñanza de la Biología.

pasado, sino como áreas dinámicas que revelan los hechos y consecuencias de estos para la humanidad sobre las perspectivas reflexiva, analítica y critica. Hay consenso, por la parte de la comunidad científica, al hecho que los aportes histórico-filosóficos en las clases puede contribuir en significativo para tornar la aprendizaje mas reflexiva, estimulante e mas coherente. En eso sentido, las publicaciones científicas deberían evidenciar la presencia de estos aportes (al menos en la perspectiva de las ciencias naturales $e$ matemática).

Teniendo en consideración el actual desarrollo mundial que ha exigido de los ciudadanos una visión cada vez más global, además, que sean capaces de analizar sus problemas, de reflexionar sobre la estrategia más adecuada y argumentar críticamente sobre las decisiones que deben ser tomadas. El cuadro educacional ha instigado a muchos estudiosos a desarrollar investigaciones con el intuito de mejorar el sistema de educación vigente.

En este sentido, la aproximación entre la Historia, la Filosofía y las Ciencias puede ofrecer a los envueltos en el proceso educacional, sea investigador, profesor o alumno, una ampliación de la visión de las Ciencias y de estas en el mundo.

\section{Referencias}

Bardin, L. (2004). Análise de conteúdo. 3. ed. Lisboa: Edições 70.

Delizoicov, D., Angotti, J. A. \& Pernambuco, M. M. (2007). Ensino de ciências: fundamentos e métodos. 2. Ed. São Paulo: Cortez.

El-Hani, C. N., Tavares, E. J. M. \& Da Rocha, P. L. B. (2004). Concepções epistemológicas de estudantes de biologia e sua transformação por uma proposta explícita de ensino sobre história e filosofia das ciências. Investigações em Ensino de Ciências, Maringá, v. 9, n. 3, p. 265-313.

Lucas, L. B. (2010). Contribuições axiológicas e epistemológicas ao ensino da teoria da evolução de Darwin. 2010. 209 fls. Dissertação (Mestrado em Ensino de Ciências e Educação Matemática) - Universidade Estadual de Londrina; Londrina. 
Bio - grafia. Escritos sobre la Biología y su Enseñanza. ISSN 2027

Edición Extraordinaria. p.p. 513 - 520

Memorias del VIII Encuentro Nacional de Experiencias en Enseñanza de la Biología y la Educación Ambiental. III Congreso Nacional de Investigación en Enseñanza de la Biología.

Luccas, S. (2004). Abordagem histórico-filosófica na educação matemática: apresentação de uma proposta pedagógica. Dissertação (Mestrado em Ensino de Ciências e Educação Matemática) - Universidade Estadual de Londrina, Londrina.

Maienschein, J. (2000). Why study history for science? Biology and Philosophy. Dordrecht, v. 15, n. 3 p. $339-348$, June.

Matthews, M. R. (1995). História, filosofia e ensino de ciências: a tendência atual de reaproximação. Caderno Catarinense de Ensino de Física, Florianópolis, v. 12, n. 3, p. 164214.

Wortmann, M. L. C. (1996). É possível articular a Epistemologia, a História da ciência e a didática no ensino científico? Episteme, Porto Alegre, v. 1, n. 1, p. 59-72. 